\title{
ON THE REFLEXIVITY OF PAIRS OF CONTRACTIONS
}

\author{
MAREK KOSIEK, ALFREDO OCTAVIO, AND MAREK PTAK
}

(Communicated by Palle E. T. Jorgensen)

\begin{abstract}
We consider pairs of commuting contractions such that the joint left essential spectrum is dominating for the algebra $H^{\infty}\left(\mathbf{D}^{2}\right)$. It is also assumed, in the first case, that one of them is $\mathbf{C}_{0}$. and the second one is absolutely continuous. In the second case, we assume that the pair is diagonally extendable. It will be shown that such pairs are reflexive.
\end{abstract}

\section{INTRODUCTION}

The dual algebras technique, after a great achievement in the problem of invariant subspaces for single operators, is being applied to pairs or $\mathrm{N}$-tuples of operators. Theorems 5.3 and 5.4 of [KP] show the reflexivity for pairs of commuting $\mathbf{C}_{0}$. or doubly commuting contractions with dominating joint left essential spectrum. The purpose of our paper is to weaken those assumptions. For the first result we need the $\mathbf{C}_{0}$. assumption for one operator only. For the second one doubly commutativity is replaced by a weaker one, diagonal extendibility (for a definition see section 4).

Throughout the paper $L(\mathscr{H})$ denotes the algebra of all linear, bounded operators in a complex separable, infinite-dimensional Hilbert space $\mathscr{H} . I_{\mathscr{H}}$ or $I$ stands for the identity in $\mathscr{H}$. By a subspace of $\mathscr{H}$, we mean a closed subspace, and by an algebra of operators - a subalgebra of $L(\mathscr{H})$ with unit $I_{\mathscr{E}}$. If $\mathscr{S}$ is a subset of $L(\mathscr{H})$, then $\mathscr{W}(\mathscr{S}), \mathscr{A}(\mathscr{S})$, and Lat $\mathscr{S}$ stand for the WOT (=weak operator topology)-closed algebra, the weak-star closed algebra, generated by $\mathscr{S}$ and $I$, and the lattice of all invariant subspaces for $\mathscr{S}$, respectively. $\operatorname{Alg} \operatorname{Lat} \mathscr{S}$ is the algebra of all operators on $\mathscr{H}$, which leave invariant all subspaces from Lat $\mathscr{S}$. An algebra $\mathscr{W}$ is called reflexive if $\mathscr{W}=$ Alg Lat $\mathscr{W}$. A family $\mathscr{S} \subset L(\mathscr{H})$ is called reflexive if so is $\mathscr{W}(\mathscr{S})$.

As usual, $A\left(\mathbf{D}^{N}\right)$ will denote the polydisc algebra, i.e., the algebra of all functions analytic on $\mathrm{D}^{N}$ and continuous on its closure, and $H^{\infty}\left(\mathbf{D}^{N}\right)$ the algebra of all bounded analytic functions on $\mathbf{D}^{N}$. A subset $E$ of $\mathbf{D}^{N}$ is called dominating for $H^{\infty}\left(\mathbf{D}^{N}\right)$ if $\sup \left\{|h(z)|: z \in \mathbf{D}^{N}\right\}=\sup \{|h(z)|: z \in E\}$ for all $h \in H^{\infty}\left(\mathbf{D}^{N}\right)$. For a complex function $f$ on $F \subset \mathbf{D}^{N}$ denote by $f^{\sim}$ the function $f^{\sim}(z)=\overline{f(\bar{z})}, \quad z \in F$.

Received by the editors April 1, 1993 and, in revised form, July 30, 1993.

1991 Mathematics Subject Classification. Primary 47A15; Secondary 47D27.

Key words and phrases. Essential spectrum, reflexive algebras, reflexive operators.

The first and the third author were partially supported by KBN grant. 
Let $T_{1}, \ldots, T_{N}$ be commuting operators on $\mathscr{H}$. Denote by $C(\mathscr{H})$ the Calkin algebra and by $\pi$ the quotient map $\pi: L(\mathscr{H}) \rightarrow C(\mathscr{H})$. Recall that the joint left essential spectrum $\sigma_{l e}\left(T_{1}, \ldots, T_{N}\right)$ of $T_{1}, \ldots, T_{N}$ is defined as the joint left spectrum of $\pi\left(T_{1}\right), \ldots, \pi\left(T_{N}\right)$. Let us recall (see [KP, Lemma 2.1]) that $\lambda=\left(\lambda_{1}, \ldots, \lambda_{N}\right) \in \sigma_{l e}\left(T_{1}, \ldots, T_{N}\right)$, if and only if there exists an orthonormal sequence $\left\{x_{n}\right\}$ such that $\lim _{n \rightarrow \infty}\left\|\left(T_{i}-\lambda_{i}\right) x_{n}\right\|=0$, for $i=$ $1, \ldots, N$. Moreover, using standard techniques we can prove

Lemma 1.1. Let $T_{1}, \ldots, T_{N}$ be commuting operators on $\mathscr{H}$; then $\lambda=$ $\left(\lambda_{1}, \ldots, \lambda_{N}\right) \in \sigma_{l e}\left(T_{1}, \ldots, T_{N}\right)$, if and only if there exists a sequence $\left\{x_{n}\right\}$ such that $x_{n} \rightarrow 0$ weakly, $\left\|x_{n}\right\|=1$, and $\lim _{n \rightarrow \infty}\left\|\left(T_{i}-\lambda_{i}\right) x_{n}\right\|=0$, for $i=1, \ldots, N$.

A contraction $T \in L(\mathscr{H})$ is absolutely continuous (a.c.) if it has no singular unitary part.

\section{REPRESENTATIONS}

Let us recall that the algebra homomorphism $\Phi: A\left(\mathbf{D}^{2}\right) \rightarrow L(\mathscr{H})$ is a representation if $\|\Phi(f)\| \leq\|f\|$ for $f \in A\left(\mathbf{D}^{2}\right)$. It is a consequence of standard techniques that for every $x, y \in \mathscr{H}$ there exists a complex, Borel, regular measure $\mu_{x, y}$ on $\overline{\mathbf{D}}^{2}$ such that $(\Phi(u) x, y)=\int u d \mu_{x, y}$ for $x, y \in \mathscr{H}, u \in A\left(\mathbf{D}^{2}\right)$. We say that $\Phi$ is absolutely continuous (a.c.), if it has a system of elementary measures $\left\{\mu_{x, y}\right\}_{x, y \in \mathscr{H}}$ such that each element of the system is absolutely continuous with respect to some (positive) representing measure $\nu_{z}, z \in \mathbf{D}^{2}$. By [G, VI.1.2, II.7.5], the above definition is equivalent to that given in [KP, Chapters 3 and 4], which uses the terminology of bands of measures.

Having the pair $\left\{T_{1}, T_{2}\right\}$ of commuting contractions in $L(\mathscr{H})$, let us construct, using Ando Theorem [SNF, I.6.4] like in [KP, Chapter 5], the representation $\Phi$ of the algebra $A\left(\mathbf{D}^{2}\right)$ generated by $\left\{T_{1}, T_{2}\right\}$, i.e., $\Phi\left(z_{i}\right)=T_{i}$, $i=1,2$. We say that the pair $\left\{T_{1}, T_{2}\right\}$ is a.c. if $\Phi$ is a.c. In the same way we can construct the representation $\Phi^{+}$of $A\left(\mathbf{D}^{2}\right)$ generated by $\left\{T_{1}^{*}, T_{2}^{*}\right\}$. Moreover we have

Lemma 2.1. (1) The representation $\Phi$ is a.c. if and only if $\Phi^{+}$is a.c.

(2) If $\left\{T_{1}, T_{2}\right\}$ is an a.c. pair of commuting contractions and $f \in H^{\infty}\left(\mathbf{D}^{2}\right)$ then for any vectors $x, y$ we have

$$
\left(f\left(T_{1}, T_{2}\right) y, x\right)=\left(y, f^{\sim}\left(T_{1}^{*}, T_{2}^{*}\right) x\right) .
$$

Proof. Let $u \in A\left(\mathbf{D}^{2}\right)$, and $\mu_{y, x}$ be an elementary measure of $\Phi$ for $y, x \in$ $\mathscr{H}$ absolutely continuous with respect to a representing measure $\nu_{z}$ for some $z \in \mathbf{D}^{2}$. It is obvious that $u^{\sim} \in A\left(\mathbf{D}^{2}\right)$.

For a complex measure $\mu$ on $\overline{\mathbf{D}}^{2}$ denote by $\mu^{\sim}$ the Borel measure $\mu^{\sim}(\cdot)=$ $\overline{\mu(\Pi(\cdot))}$, where $\Pi: z \rightarrow \bar{z}$ is a homeomorphism of $\overline{\mathbf{D}}^{2}$ onto itself. Then for $u \in A\left(\mathbf{D}^{2}\right)$ we have

$$
\begin{aligned}
\left(u\left(T_{1}^{*}, T_{2}^{*}\right) x, y\right) & =\left(x, u\left(T_{1}^{*}, T_{2}^{*}\right)^{*} y\right)=\overline{\left(x, u^{\sim}\left(T_{1}, T_{2}\right) y\right)} \\
& =\overline{\left(u^{\sim}\left(T_{1}, T_{2}\right) y, x\right)}=\int u^{\sim} d \mu_{y, x} \\
& =\int \overline{u^{\sim}} d \overline{\mu_{y, x}}=\int u d \mu_{y, x}^{\tilde{y}} .
\end{aligned}
$$


So $\eta_{x, y} \stackrel{\mathrm{df}}{=} \mu_{y, x}$ is an elementary measure of $\Phi^{+}$for vectors $x, y \in \mathscr{H}$. Since $\mu_{y, x}$ is absolutely continuous with respect to $\nu_{z}$, then $\eta_{x, y}$ is absolutely continuous with respect to $\nu_{z}^{\tilde{z}}$, and an easy calculation shows that $\nu_{z}^{\tilde{z}}$ is a representing measure for $\bar{z} \in \mathbf{D}^{2}$, which finishes the proof of (1).

Next, for (2), one can easily see that $f^{\sim} \in H^{\infty}\left(\mathbf{D}^{2}\right)$. Let, like in (1), $\mu_{y, x}$ and $\mu_{\hat{y}, x}$ be elementary measures of $\Phi$ and $\Phi^{+}$, respectively. Notice that $\sigma_{z} \stackrel{\text { df }}{=} \nu_{z}+\nu_{z}^{\sim}$ is positive and symmetric with respect to adjoint. Let $d \mu_{y, x}=$ $h_{y, x} d \sigma_{z}$. Then $d \mu_{\tilde{y}, x}^{\sim}=h_{y, x}^{\sim} d \sigma_{z}$. Hence, the following finishes the proof

$$
\begin{aligned}
\left(f\left(T_{1}, T_{2}\right) y, x\right) & =\int f d \mu_{y, x}=\int f(\lambda) h_{y, x}(\lambda) d \sigma_{z}(\lambda) \\
& =\int f(\bar{\lambda}) h_{y, x}(\bar{\lambda}) d \sigma_{z}(\bar{\lambda})=\overline{\int f \sim(\lambda) h_{\tilde{y}, x}(\lambda) d \sigma_{z}(\lambda)} \\
& =\overline{\int f \sim d \mu_{\tilde{y}, x}^{\sim}}=\overline{\left(f^{\sim}\left(T_{1}^{*}, T_{2}^{*}\right) x, y\right)}=\left(y, f^{\sim}\left(T_{1}^{*}, T_{2}^{*}\right) x\right) .
\end{aligned}
$$

Having the representation constructed above, using [K1, Proposition] (see also [K1, Sec.2], [K2, Sec.3]), we can decompose $\Phi$ and $\mathscr{H}$ into orthogonal sums as follows

$$
\Phi=\Phi_{0} \oplus \Phi_{1} \oplus \Phi_{2} \oplus \Phi_{3}, \quad \mathscr{H}=\mathscr{H}_{0} \oplus \mathscr{H}_{1} \oplus \mathscr{H}_{2} \oplus \mathscr{H}_{3},
$$

where $\Phi_{i}(i=0,1,2,3)$ is the restriction of $\Phi$ to the subspace $\mathscr{H}_{i}$ reducing all the values of $\Phi$. Moreover we get

$\Phi_{0}$ is an absolutely continuous representation,

$\left.T_{i}\right|_{\mathscr{H}_{i}}(i=1,2)$ is a unitary operator with singular spectral measure,

$\left.T_{i}\right|_{\mathscr{H}_{3}}(i=1,2)$ are unitary operators.

So we get

Lemma 2.2. If $T_{1}$ is c.n.u. and $T_{2}$ is a.c. then the pair $\left\{T_{1}, T_{2}\right\}$ is a.c.

\section{THE FIRST REFLEXIVITY RESULT}

The purpose of the section is the following

Theorem 3.1. Let $\left\{T_{1}, T_{2}\right\} \subset L(\mathscr{H})$ be a pair of commuting contractions. Assume also that $T_{1} \in \mathbf{C}_{0}$. and $T_{2}$ is absolutely continuous. If $\sigma_{l e}\left(T_{1}, T_{2}\right) \cap \mathbf{D}^{2}$ is dominating for $H^{\infty}\left(\mathbf{D}^{2}\right)$, then the algebra $\mathscr{W}\left(T_{1}, T_{2}\right)$ is reflexive.

The proof of the theorem uses the technique of dual algebras. Thus we start with some definitions and notation. The Banach space of trace class operators in $L(\mathscr{H})$ with the trace norm will be denoted by $(\tau c)$. Recall that the bilinear form $\langle A, T\rangle=\operatorname{tr}(A T), A \in L(\mathscr{H}), T \in(\tau c)$, on $L(\mathscr{H}) \times(\tau c)$ allows us to identify $L(\mathscr{H})$ with $(\tau c)^{*}$ and the weak-star topology on $L(\mathscr{H})$ under this identification coincides with the ultraweak operator topology. If $\mathscr{A}$ is any ultraweakly closed subspace of $L(\mathscr{H})$, then its preannihilator ${ }^{\perp} \mathscr{A}$ is a closed subspace of $(\tau c)$. $\mathscr{A}$ can be identified with the dual of $Q_{\mathscr{A}}=(\tau c) /{ }^{\perp} \mathscr{A}$ via the bilinear form $\langle A, T\rangle=\operatorname{tr}(A T)$ on $\mathscr{A} \times Q_{\mathscr{A}}$. The quotient norm in $Q_{\mathscr{A}}$ (so-called $Q_{\mathscr{A}}$ norm) will be denoted by $\|\cdot\|_{Q_{\alpha}}$. For simplicity we denote $Q_{T}$ and $Q_{T_{1}, T_{2}}$ 
instead of $Q_{\mathscr{A}(T)}$ and $Q_{\mathscr{A}\left(T_{1}, T_{2}\right)}$ respectively for $T, T_{1}, T_{2} \in L(\mathscr{L})$. In what follows, for $x, y \in \mathscr{H}, x \otimes y$ denotes rank one operator $(x \otimes y) z=(z, y) x$.

Proof of Theorem 3.1. The representation generated by $\left\{T_{1}, T_{2}\right\}$ is, by Lemma 2.2 , absolutely continuous. We apply the technique used in [KP, Chapter 4 and 5]. Note that [KP, Theorem 4.5] is stated for absolutely continuous representations. The careful reader can notice that all the lemmas in [KP, Chapter 4] are true not only for c.n.u. contractions but also for any pair of contractions which generate an absolutely continuous representation.

Let, as usual, [ $\left.C_{\lambda}\right], \lambda \in \mathbf{D}^{2}$, denote the element of $Q \stackrel{d f}{=} Q_{\mathscr{A}\left(T_{1}, T_{2}\right)}$ corresponding to a weak-star continuous functional of evaluation at a point $\lambda$. Notice also that like in [KP] we can obtain that $\overline{\operatorname{aco}}\left\{\left[C_{\lambda}\right]: \lambda \in \sigma_{l e}\left(T_{1}, T_{2}\right) \cap \mathbf{D}^{2}\right\}$ contains the closed unit ball in $Q$. Let us recall that a dual algebra $\mathscr{A} \subset L(\mathscr{H})$ has property $X_{0,1}$ if for all $[L] \in Q,\|[L]\| \leq 1$, we have the following:

$\left(^{*}\right)$ there exist sequences $\left\{x_{n}\right\}_{n=1}^{\infty},\left\{y_{n}\right\}_{n=1}^{\infty} \subset \mathscr{C}$ with $\left\|x_{n}\right\| \leq 1,\left\|y_{n}\right\| \leq$ 1 for $n=1,2, \ldots$ such that

$$
\begin{gathered}
\lim _{n \rightarrow \infty}\left\|\left[x_{n} \otimes y_{n}\right]-[L]\right\|_{Q}=0, \\
\lim _{n \rightarrow \infty}\left\|\left[x_{n} \otimes z\right]\right\|_{Q}=0, \quad \text { for all } z \in \mathscr{H}, \\
\lim _{n \rightarrow \infty}\left\|\left[z \otimes y_{n}\right]\right\|_{Q}=0, \quad \text { for all } z \in \mathscr{H} .
\end{gathered}
$$

It is well known [BFP] that any dual algebra with property $X_{0,1}$ is reflexive. Also the set of functionals $[L] \in Q$ satisfying $\left({ }^{*}\right)$ is absolutely convex and closed. Therefore, for the reflexivity of $\mathscr{A}\left(T_{1}, T_{2}\right)$, hence also $\mathscr{W}\left(T_{1}, T_{2}\right)$, it is enough to prove $\left({ }^{*}\right)$ for $\left[C_{\lambda}\right]$ where $\lambda \in \sigma_{l e}\left(T_{1}, T_{2}\right)$

Let $\lambda \in \sigma_{l e}\left(T_{1}, T_{2}\right)$. In [KP, Lemma 4.10, 4.11], (3.1) and (3.2) were proved with our assumptions and even without $\mathbf{C}_{0}$. , thus we need to show the statement (3.3). It will be shown below in Lemma 3.3, strengthening [KP, Lemma 5.1, 4.12].

It is an easy observation that in [R, Lemma 3.4] the assumption c.n.u. is not necessary, only the absolute continuity of $T$ is needed. Moreover, weaker assumptions for the sequence $\left\{x_{n}\right\}$ are sufficient. So we have

Lemma 3.2. Assume that $T \in L(\mathscr{H})$ is an a.c. contraction generating an isometric functional calculus. If $x_{n}$ is a sequence such that $x_{n} \rightarrow 0$ weakly, $\left\|x_{n}\right\|=1$, and $\left\|(T-\lambda) x_{n}\right\| \rightarrow 0$, then $\left\|\left[y \otimes x_{n}\right]\right\|_{Q_{T}} \rightarrow 0$ for all $y \in \mathscr{H}$.

Now we can formulate

Lemma 3.3. Assume $T_{1}, T_{2}$ generate an a.c. isometric representation and $T_{1}^{n} \rightarrow$ 0 strongly. If $x_{n}$ is a sequence such that $x_{n} \rightarrow 0$ weakly, $\left\|x_{n}\right\|=1$, and $\left\|\left(T_{2}-\lambda_{2}\right) x_{n}\right\| \rightarrow 0$, then $\left\|\left[y \otimes x_{n}\right]\right\|_{Q} \rightarrow 0$ for all $y \in \mathscr{H}$.

Proof. By [SNF, Theorem II.2.1], choosing $V_{1} \in L(\mathscr{K})$ a minimal isometric dilation of $T_{1}^{*}, V_{1}$ is a unilateral shift of uncertain multiplicity and also we have $T_{1}=V_{1}^{*} \mid \mathscr{X}$. On the other hand, by the commutant lifting theorem of Sz.-Nagy and Foias (see [SNF], [P, p. 484]) there is an operator $W_{2}$ (not necessarily isometry) preserving the norm of $T_{2}^{*}$, such that the pair $\left\{V_{1}, W_{2}\right\}$ dilates $\left\{T_{1}^{*}, T_{2}^{*}\right\}$. Let $\varepsilon>0$. Choose $M>0$ such that $\left\|\left(I-P_{\text {ker } V_{1}^{*}}\right) y\right\| \leq \frac{e}{3}$, 
and denote $y_{1}=\left(P_{\operatorname{ker} V_{1}^{* M}}\right) y, y_{2}=\left(I-P_{\operatorname{ker} V_{1}^{*}}\right) y$. By Hahn-Banach theorem, for each $n$, there is $f_{n} \in H^{\infty}\left(\mathbf{D}^{2}\right)$ such that

$$
\left\|\left[y \otimes x_{n}\right]\right\|_{Q}=\left\langle f_{n}\left(T_{1}, T_{2}\right),\left[y \otimes x_{n}\right]\right\rangle=\left(f_{n}\left(T_{1}, T_{2}\right) y, x_{n}\right),\left\|f_{n}\right\|=1 .
$$

For each $n$ we can decompose $f_{n}$ as follows

$$
f_{n}\left(z_{1}, z_{2}\right)=\sum_{k=0}^{M-1} a_{n k}\left(z_{2}\right) z_{1}^{k}+z_{1}^{M} h_{n}\left(z_{1}, z_{2}\right) .
$$

The functions $a_{n k}$ are measurable. Moreover, since $a_{n k}\left(z_{2}\right)$ is the $k$-th Fourier coefficient of $f_{n}\left(\cdot, z_{2}\right)$, then $\left|a_{n k}\left(z_{2}\right)\right| \leq 1$, for $z_{2} \in \mathbf{D}$, by (3.4). Thus $\left\|a_{n k}\right\| \leq$ 1 and consequently $\left\|h_{n}\right\| \leq M+1$. It is easy to check that the negative Fourier coefficients of every $a_{n, k}$ vanish. Hence for every $n, k$ we get $a_{n, k} \in H^{\infty}(\mathbf{D})$, and $h_{n} \in H^{\infty}\left(\mathbf{D}^{2}\right)$.

Applying the Lebesgue type decomposition (2.1) to space $\mathscr{K}$ and an easy calculation on elementary measures we get $\mathscr{H} \subset \mathscr{K}_{0}$. So, by the minimality of $V_{1}$, we have $\mathscr{K}=\mathscr{K}_{0}$, hence the pair $\left\{V_{1}, W_{2}\right\}$ is a.c. By Lemma 2.1, also $\left\{V_{1}^{*}, W_{2}^{*}\right\}$ is a.c. By [K1, Proposition], the representation generated by $T_{2}$ is a.c. and so is $W_{2}^{*}$.

The above facts give us the existence of the functional calculus for all abovementioned pairs and single operators. So, by Lemma 2.1(2) applied to the pairs $\left\{T_{1}, T_{2}\right\}$ and $\left\{V_{1}^{*}, W_{2}^{*}\right\}$, we have

$$
\begin{aligned}
\left\|\left[y \otimes x_{n}\right]\right\|_{Q}= & \left|\left(f_{n}\left(T_{1}, T_{2}\right) y, x_{n}\right)\right|=\left|\left(y, f_{n}^{\sim}\left(T_{1}^{*}, T_{2}^{*}\right) x_{n}\right)\right| \\
\leq & \left|\left(y, f_{n}^{\sim}\left(V_{1}, W_{2}\right) x_{n}\right)\right|=\left|\left(f_{n}\left(V_{1}^{*}, W_{2}^{*}\right) y, x_{n}\right)\right| \\
\leq & \left|\left(f_{n}\left(V_{1}^{*}, W_{2}^{*}\right) y_{1}, x_{n}\right)\right|+\left|\left(f_{n}\left(V_{1}^{*}, W_{2}^{*}\right) y_{2}, x_{n}\right)\right| \\
\leq & \sum_{k=0}^{M-1}\left|\left(a_{n k}\left(W_{2}^{*}\right) V_{1}^{* k} y_{1}, x_{n}\right)\right| \\
& +\left|\left(V_{1}^{* M} h_{n}\left(V_{1}^{*}, W_{2}^{*}\right) y_{1}, x_{n}\right)\right|+\left\|f_{n}\right\|\left\|y_{2}\right\|\left\|x_{n}\right\| .
\end{aligned}
$$

The vector $y_{1}$ was defined such that $V_{1}^{* M} y_{1}=0$, thus the second component is equal 0 . Observe that for all $k$

$$
\left|\left(a_{n k}\left(W_{2}^{*}\right) V_{1}^{* k} y_{1}, x_{n}\right)\right| \leq\left\|\left[V_{1}^{* k} y_{1} \otimes x_{n}\right]\right\|_{Q_{w_{2}^{*}}} .
$$

On the other hand, by [SNF, Theorem II.2.3], $W_{2}^{*}$ is an extension of $T_{2}$, thus not only $\left\|\left(W_{2}^{*}-\lambda_{2}\right) x_{n}\right\| \rightarrow 0$, but also $W_{2}^{*}$ generates an isometric representation, since $T_{2}$ does. Hence, Lemma 3.2 shows that $\left\|\left[V_{1}^{* k} y_{1} \otimes x_{n}\right]\right\|_{Q_{2}^{*}} \rightarrow$ $0(n \rightarrow \infty)$. Hence we can choose $n_{0}$ such that for all $n>n_{0}$ we have $\left\|\left[V_{1}^{* k} y_{1} \otimes x_{n}\right]\right\|_{Q_{w_{2}^{*}}} \leq \frac{\varepsilon}{3 M}$ for $k=0,1, \ldots, M-1$. Coming back to the estimation of $\left\|\left[y \otimes x_{n}\right]\right\|_{Q}$, using (3.6) and the estimation of $\left\|y_{2}\right\|$, we obtain for $n>n_{0}$

$$
\left\|\left[y \otimes x_{n}\right]\right\|_{Q} \leq \sum_{k=0}^{M-1}\left\|\left[V_{1}^{* k} y_{1} \otimes x_{n}\right]\right\|_{Q_{w_{2}^{*}}}+\left\|f_{n}\right\|\left\|y_{2}\right\|\left\|x_{n}\right\| \leq \varepsilon .
$$

The proof of the lemma is finished. 
Let us notice in the end of the section that by taking adjoints using Theorem 3.1 we can obtain reflexivity assuming the dominating property for the right essential joint spectrum and $\mathbf{C}_{._{0}}$ assumption for one operator.

\section{THE SECOND REFLEXIVITY RESULT}

This section deals with pairs of operators which extend the case of doubly commuting operators. For any pair $\left\{T_{1}, T_{2}\right\}$ of commuting contractions we can construct a minimal isometric dilation $\left\{V_{1}, V_{2}\right\}$ of the pair $\left\{T_{1}^{*}, T_{2}^{*}\right\}$ (see [SNF]). One can see that $V_{i}^{*}$ is a coisometric extension of $T_{i}, i=1,2$. We call $\left\{V_{1}^{*}, V_{2}^{*}\right\}$ a joint coisometric extension of $\left\{T_{1}, T_{2}\right\}$. It can be seen that it is minimal in standard meaning, for the details see [O]. We say that a pair $\left\{T_{1}, T_{2}\right\} \subset L(\mathscr{H})$ is diagonally extendable if there exist a Hilbert space $\mathscr{K} \supset \mathscr{H}$ and a minimal joint coisometric extension $\left\{B_{1}, B_{2}\right\} \subset L(\mathscr{H})$ of $\left\{T_{1}, T_{2}\right\}$ such that, for either $j=1$ or $j=2$, if $\mathscr{K}$ is decomposed as $\mathscr{K}=\mathscr{S}_{j} \oplus \mathscr{R}_{j}$, relative to which the matrix for $B_{j}$ has the form

$$
B_{j}=\left(\begin{array}{cc}
S_{j}^{*} & 0 \\
0 & R_{j}
\end{array}\right)
$$

where $S_{j}^{*} \in L\left(\mathscr{S}_{j}\right)$ is a (unilateral) backward shift and $R_{j} \in L\left(\mathscr{R}_{j}\right)$ is a unitary operator, then the matrix for $B_{k}$, for $k \neq j$, relative to the decomposition $\mathscr{K}=\mathscr{S}_{j} \oplus \mathscr{R}_{j}$ has the form

$$
B_{k}=\left(\begin{array}{cc}
A_{s} & 0 \\
0 & A_{r}
\end{array}\right),
$$

for some $A_{s} \in L\left(\mathscr{S}_{j}\right)$ and $A_{r} \in L\left(\mathscr{R}_{j}\right)$.

Let us recall the result of [O, Theorem 2.5] and [S, Lemma 1] as

Proposition 4.1. With the above notation, a pair $\left\{T_{1}, T_{2}\right\} \subset L(\mathscr{H})$ is diagonally extendable if any of the following conditions holds:

(a) $R_{1}$ has no part of uniform multiplicity $\aleph_{0}$,

(b) $R_{2}$ has no part of uniform multiplicity $\aleph_{0}$,

(c) $T_{1}$ and $T_{2}$ doubly commute.

The main result of this section is the following

Theorem 4.2. Let $\left\{T_{1}, T_{2}\right\} \subset L(\mathscr{H})$ be a pair of a.c. commuting contractions. Assume also that $\left\{T_{1}, T_{2}\right\} \subset L(\mathscr{H})$ is diagonally extendable. If $\sigma_{l e}\left(T_{1}, T_{2}\right) \cap \mathbf{D}^{2}$ is dominating for $H^{\infty}\left(\mathbf{D}^{2}\right)$, then the algebra $\mathscr{W}\left(T_{1}, T_{2}\right)$ is reflexive.

The proof is based on the same considerations as a proof of Theorem 3.1, using Lemma 4.3, given below, instead of Lemma 3.3.

Lemma 4.3. Let $\left\{T_{1}, T_{2}\right\}$ be a diagonally extendable pair of contractions generating an a.c. isometric representation. If $x_{n}$ is a sequence such that $x_{n} \rightarrow 0$ weakly, $\left\|x_{n}\right\|=1$, and $\left\|\left(T_{i}-\lambda_{i}\right) x_{n}\right\| \rightarrow 0$ for $i=1,2$, then $\left\|\left[y \otimes x_{n}\right]\right\|_{Q} \rightarrow 0$ for all $y \in \mathscr{H}$.

Proof. Without loss of generality, we assume that the space $\mathscr{K} \supset \mathscr{H}$ and operator $B_{i} \in L(\mathscr{K})$ extend $T_{i}$ for $i=1,2$. Moreover, $B_{1}$ is a coisimetry, $\mathscr{K}=\mathscr{S}_{1} \oplus \mathscr{R}_{1}$,

$$
B_{1}=\left(\begin{array}{cc}
S_{1}^{*} & 0 \\
0 & R_{1}
\end{array}\right)
$$


where $S_{1}^{*} \in L\left(\mathscr{S}_{1}\right)$ is a (unilateral) backward shift and $R_{1} \in L\left(\mathscr{R}_{1}\right)$ is a unitary operator, and $B_{2}$ has the form

$$
B_{2}=\left(\begin{array}{cc}
A_{s} & 0 \\
0 & A_{r}
\end{array}\right),
$$

for some $A_{s} \in L\left(\mathscr{S}_{1}\right)$ and $A_{r} \in L\left(\mathscr{R}_{1}\right)$.

By decomposition (2.1) and the minimality of $\left\{B_{1}, B_{2}\right\}$ we conclude similarly as in the proof of Lemma 3.3 that $\left\{B_{1}, B_{2}\right\}$ is an a.c. pair. It also generates an isometric functional as an extension of the pair $\left\{T_{1}, T_{2}\right\}$. Thus we have

$$
\begin{aligned}
\left\|\left[y \otimes x_{n}\right]\right\|_{Q} & =\sup \left\{\left|\left\langle h\left(T_{1}, T_{2}\right) y, x_{n}\right\rangle\right|: h \in H^{\infty}\left(\mathbf{D}^{2}\right),\|h\|_{\infty}=1\right\} \\
& =\sup \left\{\left|\left\langle h\left(B_{1}, B_{2}\right) y, x_{n}\right\rangle\right|: h \in H^{\infty}\left(\mathbf{D}^{2}\right),\|h\|_{\infty}=1\right\} \\
& =\left\|\left[y \otimes x_{n}\right]\right\|_{Q_{B_{1}, B_{2}}} .
\end{aligned}
$$

So we need to show that the last tends to zero.

For any contraction $T$ and $\lambda \in \mathbf{D}$, let $T^{\lambda}$ denote the operator $(T-\lambda) \cdot$ $(I-\bar{\lambda} T)^{-1}$. An easy calculation, based on [SNF], shows that the decomposition $\mathscr{K}=\mathscr{S}_{1} \oplus \mathscr{R}_{1}$ is also a decomposition of $\left(B_{1}^{*}\right)^{\lambda_{1}}$ for a pure isometry and a unitary part. Let us note that $\mathscr{S}_{1}, \mathscr{R}_{1}$ reduce $B_{2}^{\lambda_{2}}$. Moreover, $B_{i}^{\lambda_{i}}$ is a coisometric extension of $T_{i}^{\lambda_{i}}$, for $i=1,2$. Let $x_{n}=x_{n}^{s} \oplus x_{n}^{r}, y=y^{s} \oplus y^{r}$ be the orthogonal sums with respect to the decomposition $\mathscr{K}=\mathscr{S}_{1} \oplus \mathscr{R}_{1}$. Since $\left\|\left(T_{1}-\lambda_{1}\right) x_{n}\right\| \rightarrow 0$, thus $\left\|T_{1}^{\lambda_{1}} x_{n}\right\| \rightarrow 0$. One can easily see that

$$
\begin{aligned}
\left\|x_{n}^{r}\right\| & \leq\left\|x_{n}-P_{\operatorname{Ker} B_{1}^{\lambda_{1}}} x_{n}\right\|=\left\|\left(I-P_{\operatorname{Ker} B_{1}^{\lambda_{1}}}\right) x_{n}\right\| \\
& =\left\|\left(B_{1}^{*}\right)^{\lambda_{1}} B_{1}^{\lambda_{1}} x_{n}\right\|=\left\|T_{1}^{\lambda_{1}} x_{n}\right\| \rightarrow 0,
\end{aligned}
$$

Thus,

$$
\begin{aligned}
\|[y \otimes & \left.x_{n}\right] \|_{Q_{B_{1}, B_{2}}} \\
= & \sup \left\{\left|\left\langle h\left(B_{1}, B_{2}\right) y, x_{n}\right\rangle\right|: h \in H^{\infty}\left(\mathbf{D}^{2}\right),\|h\|_{\infty}=1\right\} \\
\leq & \sup \left\{\left|\left\langle h\left(S_{1}^{*}, A_{s}\right) y^{s}, x_{n}^{s}\right\rangle\right|: h \in H^{\infty}\left(\mathbf{D}^{2}\right),\|h\|_{\infty}=1\right\} \\
& +\sup \left\{\left|\left\langle h\left(R_{1}, A_{r}\right) y^{r}, x_{n}^{r}\right\rangle\right|: h \in H^{\infty}\left(\mathbf{D}^{2}\right),\|h\|_{\infty}=1\right\} \\
\leq & \left\|\left[y^{s} \otimes x_{n}^{s}\right]\right\|_{Q_{S_{1}^{*}, A_{s}}}+\left\|y^{r}\right\|\left\|x_{n}^{r}\right\| .
\end{aligned}
$$

The second component converges to 0 . To proof that the first one converges to 0 , it is enough to show that the pair $\left\{S_{1}^{*}, A_{s}\right\}$ and sequence $z_{n}=x_{n}^{s} /\left\|x_{n}^{s}\right\|$ fulfill the assumption of Lemma 3.3. The operator $S_{1}^{*}$ converges strongly to 0 as a backward shift. Since $B_{2}$ is an extension of $T_{2}$, thus $\left\|\left(B_{2}-\lambda_{2}\right) x_{n}\right\| \rightarrow 0$. Since $\left\|\left(B_{2}-\lambda_{2}\right) x_{n}\right\|^{2}=\left\|\left(A_{s}-\lambda_{2}\right) x_{n}^{s}\right\|^{2}+\left\|\left(A_{r}-\lambda_{2}\right) x_{n}^{r}\right\|^{2}$, thus $\left\|\left(A_{s}-\lambda_{2}\right) x_{n}^{s}\right\| \rightarrow 0$ and $\left\|\left(A_{s}-\lambda_{2}\right) z_{n}\right\| \rightarrow 0$, since $\left\|x_{n}^{s}\right\| \rightarrow 1$. Hence, we can finish the proof of Lemma and Theorem 4.1 with the following

Lemma 4.4. The pair $\left\{S_{1}^{*}, A_{s}\right\}$ generates a.c. isometric representation.

Proof. To see that the pair $\left\{S_{1}^{*}, A_{s}\right\}$ generates an a.c. representation it is enough to notice that it is a restriction of the pair $\left\{B_{1}, B_{2}\right\}$, which is a.c. pair, and then make an easy calculation on elementary measures. Now we prove that $\sigma_{l e}\left(T_{1}, T_{2}\right) \cap \mathbf{D}^{2} \subset \sigma_{l e}\left(S_{1}^{*}, A_{s}\right) \cap \mathbf{D}^{2}$. Let $\left(\lambda_{1}, \lambda_{2}\right) \in \sigma_{l e}\left(T_{1}, T_{2}\right) \cap \mathbf{D}^{2}$, and $\left\{x_{n}\right\}$ is an orthonormal sequence such that $\lim _{n \rightarrow \infty}\left\|\left(T_{i}-\lambda_{i}\right) x_{n}\right\|=0$, for $i=1,2$. 
Hence, $\lim _{n \rightarrow \infty}\left\|\left(B_{i}-\lambda_{i}\right) x_{n}\right\|=0$, for $i=1,2$, too. Let $x_{n}=x_{n}^{s} \oplus x_{n}^{r}$ is the orthogonal sum with respect to the decomposition $\mathscr{K}=\mathscr{S}_{1} \oplus \mathscr{R}_{1}$. We can prove as above that $\left\|\left(A_{s}-\lambda_{2}\right) x_{n}^{s}\right\| \rightarrow 0$ and in the same way that $\left\|\left(S_{1}^{*}-\lambda_{1}\right) x_{n}^{s}\right\| \rightarrow 0$. Like in (4.1) it can be shown that $\left\|x_{n}^{r}\right\| \rightarrow 0$, hence $\left\|x_{n}^{s}\right\| \rightarrow 1$. Let $z_{n}=$ $x_{n}^{s} /\left\|x_{n}^{s}\right\|$. It is easy to check that $\left\|\left(A_{s}-\lambda_{2}\right) z_{n}\right\| \rightarrow 0$ and $\left\|\left(S_{1}^{*}-\lambda_{1}\right) z_{n}\right\| \rightarrow 0$. Thus $\left(\lambda_{1}, \lambda_{2}\right) \in \sigma_{l e}\left(S_{1}^{*}, A_{s}\right) \cap \mathbf{D}^{2}$ by Lemma 1.1. Hence, if $\sigma_{l e}\left(T_{1}, T_{2}\right) \cap \mathbf{D}^{2}$ is dominating for $H^{\infty}\left(\mathrm{D}^{2}\right)$, then $\sigma_{l e}\left(S_{1}^{*}, A_{s}\right) \cap \mathrm{D}^{2}$ also does. Hence, by [KP, Lemmas 4.1 and 4.4], the proof of the lemma is finished.

Remark 4.5. If a pair of operators fulfills assumptions of Theorem 3.1 or 4.2 , then it has a common nontrivial invariant subspace.

\section{REFERENCES}

[BFP] H. Bercovici, C. Foias, C. Pearcy, Dual algebras with applications to invariant subspaces and dilation theory, CBMS-NSF Regional Conf. Ser. in Appl. Math., vol. 56, Amer. Math. Soc., Providence, RI, 1985.

[G] T. W. Gamelin, Uniform algebras, Prentice-Hall, Englewood Cliffs, NJ, 1969.

[K1] M. Kosiek, Lebesgue-type decomposition of a pair of commuting Hilbert space operators, Bull. Acad. Polon. Sci. Ser. Sci. Math 27 (1979), 583-589.

[K2] - Representation generated by a finite number of Hilbert space operators, Ann. Polon. Math. 44 (1984), 309-315.

[KP] M. Kosiek and M. Ptak, Reflexivity of N-tuples of contractions with rich joint left essential spectrum, Integral Equations Operator Theory 13 (1990), 395-420.

[O] A. Octavio, Coisometric extension and functional calculus for pairs of commuting contractions (to appear).

[P] S. Parrot, Unitary dilation for commuting contractions, Pacific J. Math. 34 (1970), 481-490.

[R] G. Robel, On the structure of (BCP)-operators and related algebras, J. Operator Theory 12 (1984), 23-45.

[S] M. Słocinski, Isometric dilation of doubly commuting contractions and related models, Bull. Acad. Sci. Pol. 25 (1977), 1233-1242.

[SNF] B. Sz.-Nagy and C. Foias,, Analyse harmonique des operateurs de l'espace de Hilbert, Masson et Cie, Academiai Kiado, 1967.

Institute of Mathematics, Jagiellonian University, Reymonta 4, 30-059 Kraków, Poland

E-mail address: mko@im.uj.edu.pl

Departamento de Matematicas, I. V. I. C., Caracas, Venezuela

E-mail address: aoctavio@ivic.ivic.ve

INSTitute of Mathematics, University of Agriculture, Królewska 6, 30-045 Kraków, Poland

E-mail address: rmptakecyf-kr.edu.pl 